\title{
Machine Vision and Image Processing for Automated Cell Injection
}

\author{
W.H. Wang, Member, IEEE, D. Hewett, C.E. Hann, J.G. Chase and X.Q. Chen Senior Member, IEEE
}

\begin{abstract}
This paper presents image processing algorithms for cell structure recognition, which provides the desired deposition destinations without human interference for an automated cell injection system. Adherent cells (endothelial cells) are the main focus. The surface and shadow information of the nucleoli of endothelial cells is used to extract their locations, which subsequently produce a desired deposition destination inside the nucleus by Delaunay triangulation. 436 nucleoli were $92 \%$ correctly recognized, paving the way for an automated adherent cell injection system to be developed.
\end{abstract}

\section{INTRODUCTION}

耳OR investigating specific cellular responses, drug 1 compounds and biomolecules need to be delivered into individual biological cells in a precise and dose-controllable manner. For instance, four genes, OCT4, SOX2, NANOG, and LIN28, introduced into human somatic cells, were found to be sufficient to reprogram those cells to pluripotent stem cells that exhibit the essential characteristics of embryonic stem cells [1]. Apart from genes, the nucleic acid based molecules, such as short interfering RNA (siRNA) and antisense oligonucleotides (AS-ON) [2], which inhibit disease or cancer-related gene expression, need to be delivered into cells to evaluate their effectiveness.

Many technologies have been developed for cellular material delivery, including ultrasound technique [3], electroporation [4], nanovector-based delivery $[5,6]$, and mechanical microinjection [7]. Among these techniques, microinjection is effective in delivering macromolecules of a soluble or insoluble compound into almost any cell type with high rates of cell viability [8], involving a glass micropipette, which is manipulated to poke cells and deposit materials inside. In the laboratory, the state-of-the-art microinjection is typically conducted manually and the operator has to stare at images either through the microscope objectives or displayed on a monitor, while moving the micropipette tip to the desired deposition destination. In addition, this destination is selected manually and varies among cells, requiring further significant operator input and experience. The laborious task of current manual injection thus easily causes fatigue in injection technicians, and hinders performance consistency and success rates [9-12]. Hence, there is a significant and growing demand for fully automated, high-throughput injection systems.

Efforts in automating cell injection have been continuous, resulting in a visually servoed system [7], semi-automated

Wang, Hewett, Hann, Chase and Chen are with the Dept. of Mechanical Engineering, University of Canterbury, Private Bag 4800, Christchurch 8020. New Zealand (email: wenhui.wang@ canterbury.ac.nz). systems [13-15] and many tele-operated systems [16-18], to name just a few. These systems are limited in throughput and reproducibility because operator input (e.g., locating features and destinations) or operator involvement (e.g., switching from one cell to another or injector alignment) is still required. A major holdup is the need for operator input and oversight in selecting or identifying specific target locations or/and cells.

Therefore, to fully automate a cell injection system, an essential component is machine vision. Machine vision is expected to serve two main purposes:

i) to replace an operator to recognize the cell structures, and thus generate the deposition destinations; and

ii) to provide the highly accurate sensor input to control the micropipette tip, which is moved to the deposition destinations to conduct the injection. Especially, to align the micropipette tip with the cells in height.

Correspondingly, the challenges for machine vision in the context of cell injection lie in two main aspects:

i) robust recognition of the cell structures regardless of surrounding environments such as the illumination, cell medium, cell holding device, and variations for cell size and morphology; and

ii) precise location of the micropipette tip in height with respect to the deposition destinations. Once these two open challenges are addressed it will become possible to integrate machine vision with a motorized stage or micromanipulator with a high precision positioning resolution (e.g., 40nm) to construct a fully automated cell injection system.

Recently, a first-of-its-kind fully automated zebrafish embryo injection system was developed [19]. The system is currently being extended to handle adherent cell injection. As the second challenge has been addressed and reported by Wang et al [20], the focus in this paper is on the image processing for cell structure recognition and the determination of the deposition destinations based on the recognized structures.

In this research cells may be broadly classified as suspended cells (e.g., embryos/oocytes) and adherent cells (e.g., HeLa cells, fibroblasts, and endothelial cells). Here, endothelial cells are chosen for injection study. Endothelial cells line the entire circulatory system from the heart to the smallest capillary, playing important roles in the vascular system. In vitro, sticking to the Petri dish surface, endothelial cells do not require immobilization efforts. They are also highly irregular in morphology, which makes robust pattern recognition potentially very challenging.

The paper is organized as follows. First, the overall 
structure of a cell injection system involving machine vision for the adherent cells is introduced. Next, the image processing method for endothelial cells is presented. The experimental results for structure recognition are then demonstrated.

\section{STRUCTURE OF A CELL INJECTION SYSTEM}

Fig. 1 shows the general structure for an adherent cell injection system. Two motorized positioners 1,2 , such as multi-DOF motorized positioning stages or microrobots/micromanipulators, control the motion of micropipette. A control software unit runs on the host computer for motion control and image processing. Position control devices connected to, or mounted on, the host computer physically provide control signals to the two positioners and the pressure unit.

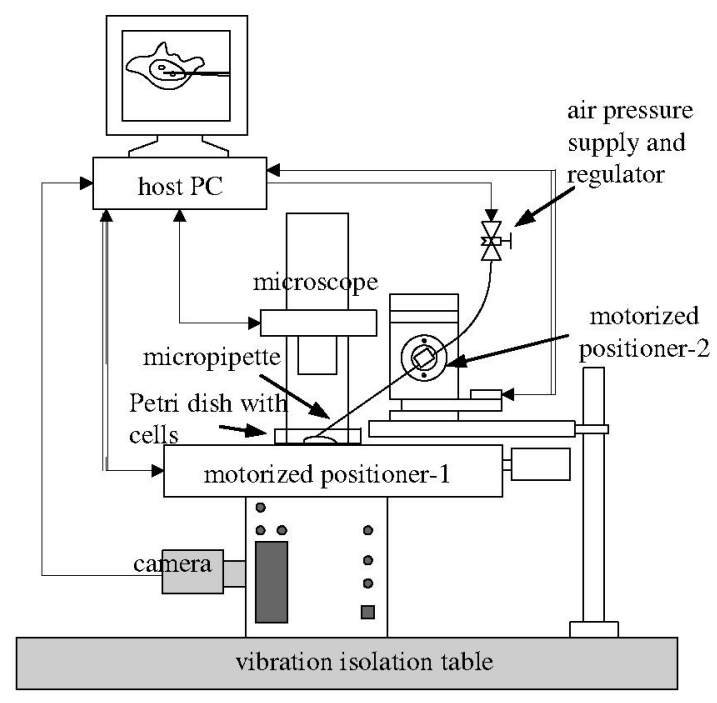

Fig. 1. Endothelial cell injection with glass micropipette.

An injection micropipette (glass capillary or microfabricated needle) is attached to the second positioner 2 , and an inverted microscope is used to provide long working distance (e.g., $>60 \mathrm{~mm}$ ) under a high magnification $(40 \times)$ for the small adherent cells. The computer-controlled pressure unit is responsible to apply pressure pulses to the micropipette for material injection. All the units except the host computer and pressure unit are mounted on a vibration isolation table to minimize vibration. Using the phase contrast or relief contract condenser, a pseudo-3D image of the cells is obtained (e.g., Fig. 2) and processed for endothelial cell structure recognition.

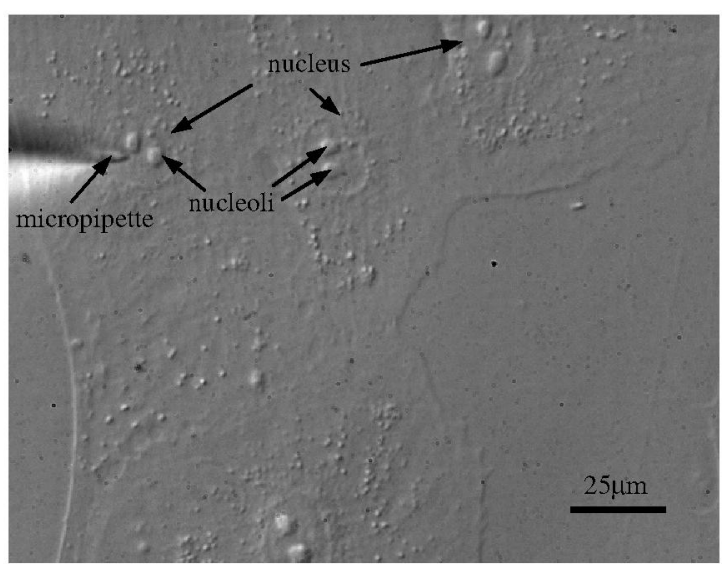

Fig. 2. Endothelial cell injection with glass micropipette.

\section{IMAGE PROCESSING FOR ENDOTHELIAL CELL STRUCTURE RECOGNITION}

In extending the zebrafish embryo injection system (Wang et al, 2007a) to adherent endothelial cells, the first task is to process the pseudo-3D images (Fig. 2) obtained for endothelial cells under the experimental setup shown in Fig. 1. For biological purposes, injection of materials should occur inside the nucleus, which carries the genetic information. Ideally, recognition of the nucleus boundary will allow the determination of a deposition destination. However, the nucleus boundary is either completely invisible or partially visible in the harsh pseudo-3D images.

Alternatively, the nucleoli inside the nucleus are more recognizable and provide hook points in the image for automatically detecting other regions for injection. Therefore, this research concentrates primarily on automatically detecting nucleoli. A deposition destination inside the nucleus may then be found based on recognized nucleoli for use in a fully automated adherent cell injection system under development. This approach will present injection errors, but eliminate the very difficult problem of identifying non-convex nucleus boundaries for these cells.

Image gradient-based methods, such as the Canny edge detector [21, 22] or snake tracking [23] are not utilized to recognize the nucleoli. In particular, gradient approaches can be sensitive to noise. In contrast, snake tracking is accurate, but computationally expensive for a lot of nucleoli presenting in one image and thus not suitable for the real-time application considered in this paper. More specifically, the harsh images present a significant number of image gradients and any type of generic edge detector would detect many spurious points, making it difficult to reliably find the required nucleoli. Furthermore, many of these feature 
detection algorithms are developed for general images, and are thus not well suited to exploiting the specific individual characteristics and knowledge of a specific application without significant tuning. Thus, the algorithm developed here is based on the surface and shadow information of nucleoli, which provides a robust approach for this particular machine vision scenario.

\section{AUTOMATED NUCLEOLI IDENTIFICATION}

An example of a typical image that is required to be processed is given in Fig 3.

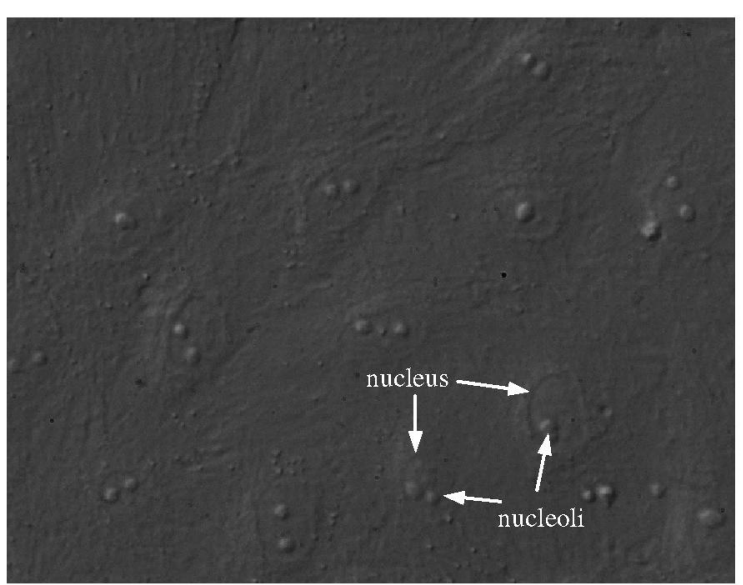

Fig. 3. An example image required to be processed. The goal is to find locations for injection by the micro pipette.

To allow consistent thresholding, the first step is to normalize the image. This normalization scales all the pixels so that they have an intensity between 0 and 255 . Let be a given pixel intensity, and and the minimum and maximum intensities in the raw image. The scaled intensity, denoted by $\bar{I}$, is defined:

$$
\bar{I}=255\left(\frac{I-I_{\min }}{I_{\max }-I_{\min }}\right)
$$

The normalized image is shown in Fig. 4.

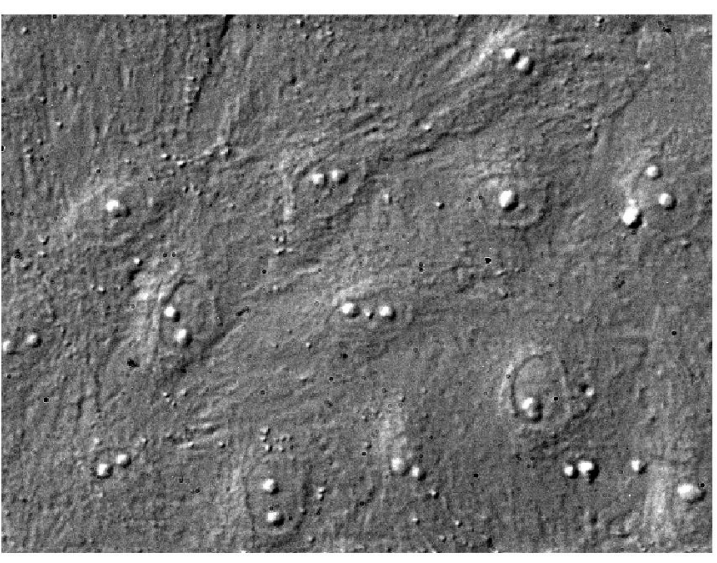

Fig. 4. Normalizing the image of Fig 3.

The nucleoi have been found to consistently contain a "light region" within them which allows a simple initial detection by using a threshold on the normalized image. Hence, the next step is to choose a threshold and remove all pixels with an intensity. The remaining points after this threshold will be the "light region" in each nucleoi. This step gives a rough detection of nucleoi, but typically contains false points. Note that the initial thresholding only gives a set of 1's and 0's in the intensity matrix, where a "1" corresponds to a potential pixel on a nucleoi. Pixels that are joined with each other (by one pixel) are then grouped together as one component. Thus a set of connected components or "blobs" corresponding to the "light region" on the image are characterized. Note that this task is non-trivial to perform and a naive approach can easily result in significant computation. However, there are already good efficient algorithms available in the literature, for example commands in Matlab [24], which very quickly finds connected components in any binary image.

The false points or "blobs" are ruled out by taking advantage of a feature specific to the images in this application. The nucleoi typically protrude out of the cell, so that they cast a shadow to the right and downwards as can be seen in Figures 3 and 4 . This feature is consistent over all images as there is a constant light source from the top left corner of the image. Hence a major discriminating feature which is used to confirm nucleoi, is whether they have a group of dark pixels to the right and downwards corresponding to the shadow. The group of dark pixels are found by first looking for all pixels on the normalized image which have an intensity less than a given amount, which is chosen to be 50. The dark pixels are then grouped into connected components. The connected components which correspond to a small neighbourhood (within 2 pixels) of the nucleoi are chosen, and thus represent the nucleoi's shadow.

For a candidate shadow to be the 'real' shadow, three criteria need to be met: 
i) It is in the right place in relation to the surface within a tolerance. The "right place" is defined as more than $80 \%$ of the shadow lying in the bottom right hand corner region defined as "R" in Fig. 5;

ii) The shadow and the surface are about the same size within a factor of 2; and

iii) The shadow is not simply a vertical or horizontal line.

A surface and shadow are paired according to the distance between them being less than 10 pixels, which can be easily adapted experimentally in the field of view of camera used. Given a pair of these two components corresponding to the surface and shadow of a nucleolus, a boundary surrounding them is obtained by computing the convex hull, which is used as an approximation to the boundary of the nucleoli. This approximation is reasonable as the shadow increases the size of the nucleoli by typically only about $10 \%$. An approximation to the boundary of each nucleolus allows a further set of criteria used to abandon spurious nucleoli. More specifically, these criteria are:

i) The enclosed area of the convex hull, measured by the number of pixels of each candidate nucleolus, must be sufficiently large but not too large, with the constraint: $80<$ Area<500 pixels; and

ii) The smallest distance in the $x$ or $y$ direction is no smaller than one tenth of the larger distance in the opposite direction.

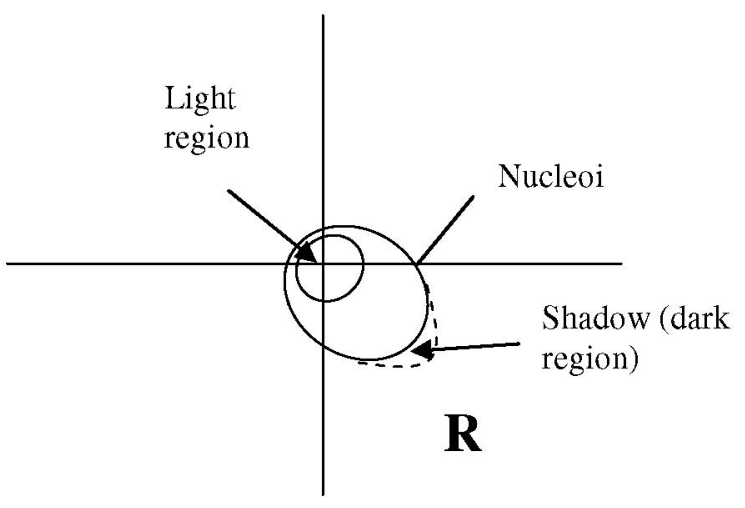

Fig 5: Defining the shadow and position of the nucleoi as the region " $\mathrm{R}$ ".

Overall, this method is reasonably general. The thresholds and tolerances can be readily adapted to similar cases with different experimental approaches. Similarly, for the location or size of shadow features based on lighting, which, if perfect, requires only thresholding the lighted region.

\section{DETERMINATION OF DEPOSITION DESTINATIONS}

The nucleoli typically occur in pairs in the nucleus for these cells. Therefore, a deposition destination that is guaranteed to lie within the nucleus is between close pairs of identified nucleoli. Two nucleoli are detected as being part of the nucleus when they lie within a specified tolerance of each other. This tolerance may be readily empirically determined based on the type of cells. The procedure is described as follows:

i) The centre of each identified nucleolus is first found;

ii) A Delaunay triangulation is then performed on the centre points of each nucleolus. This approach enables very fast and efficient calculation of the closest point to each nucleolus;

iii) The distance between the centres of each identified nucleolus is computed and pairs that lie within a specified tolerance are characterized; and

iv) Denote the line connecting the centres by $L_{1}$. The deposition destination is determined to be half way between the two points defined by the intersection of $L_{1}$ and the boundaries of each nucleolus.

\section{RESULTS AND DISCUSSION}

The first step of the algorithm after loading the images is to perform the normalization procedure of Equation (1). The result of normalizing Fig 3 is shown in Fig 4. As can be seen in Fig 4, the normalization of the images increases the contrast and makes the nucleoi, significantly stand out as white patches on the image. This feature enables the nucleoi to be easily separated from the background. Based on experiment, a threshold of 200 on the normalized image was found to consistently identify nucleoi on all images, with only a small number of false points. Fig 6 shows the result of calculating the "light regions" from Fig 4 which have a intensity greater than 200 .

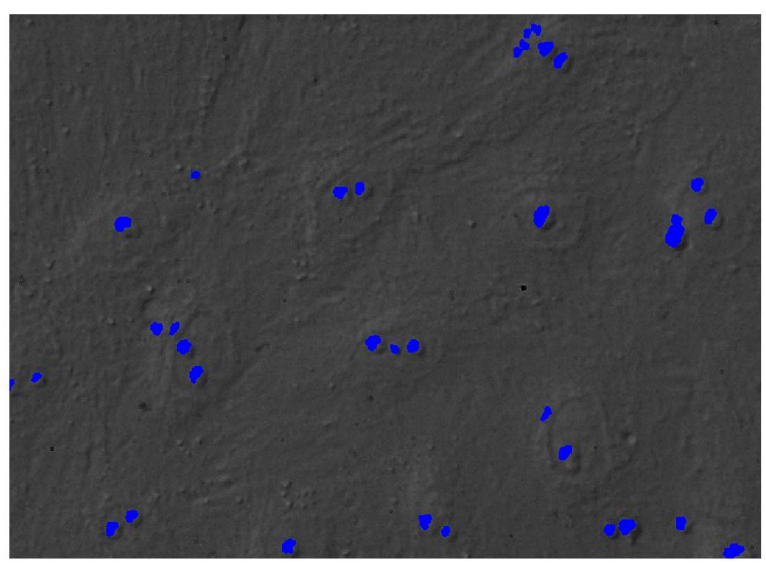

Fig 6: Thresholding Fig 4 to locate all potential nucleoi 
The next step is to look for shadows with an intensity below the threshold of 50, to the right and downwards from the "light regions" as described in the algorithm above. The end result is given in Fig 7, which shows all the "light regions" in blue and the shadows or "dark regions" in red.

Note that Fig. 7 contains some false nucleoi. Applying the automated nucleoi finding algorithm of section IV rules out these false nucleoi leaving the required true nucleoi. Fig 8 shows the final result of the detected nucleoi for the image of Fig 3. This algorithm was then applied to all the images in the data base and the results were checked with manual analysis by eye. The results are summarized as follows.

- Out of all the nucleoli that were identified, successful nucleoli identification corresponded to $92 \%$.

- Percentage of all nucleous that were identified was $86 \%$ (that is, the missed nucleoli were $14 \%$ )

- The false positives were $6 \%$

To demonstrate the algorithm of detecting the injection site, the image from Fig 3 is again used. The Delaunay triangulation from the algorithm of section $\mathrm{V}$ is shown in Fig 8 , and the injection sites are marked with a cross in Fig 9.

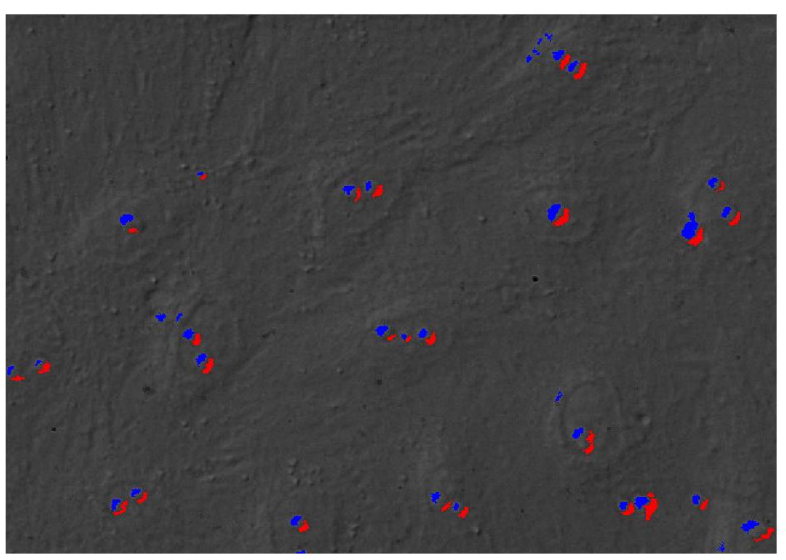

Fig 7: The end result after applying the algorithm for finding the "light regions" and "dark regions". The "light regions" are in blue and the "dark regions" are in red.

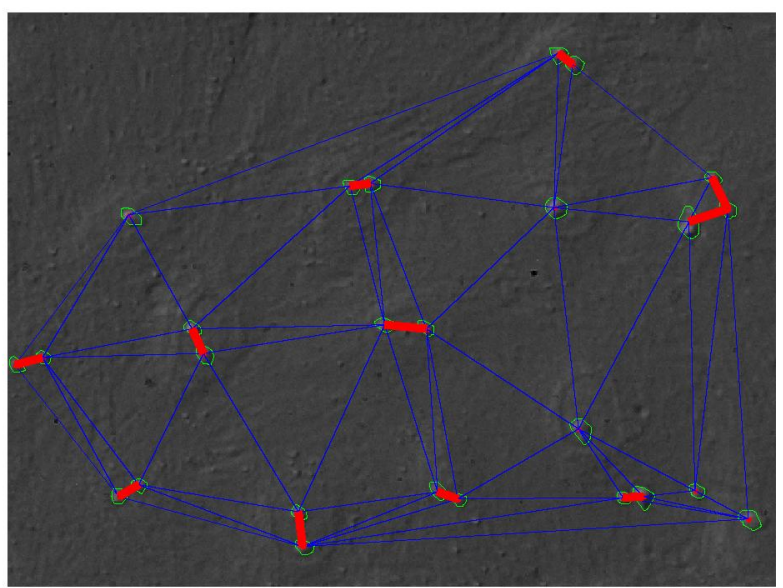

Fig 8: The result of applying Delaunay triangulation to the identified nucleoi. Close pairs are shown by red lines, and the Delaunay triangulation is plotted in blue

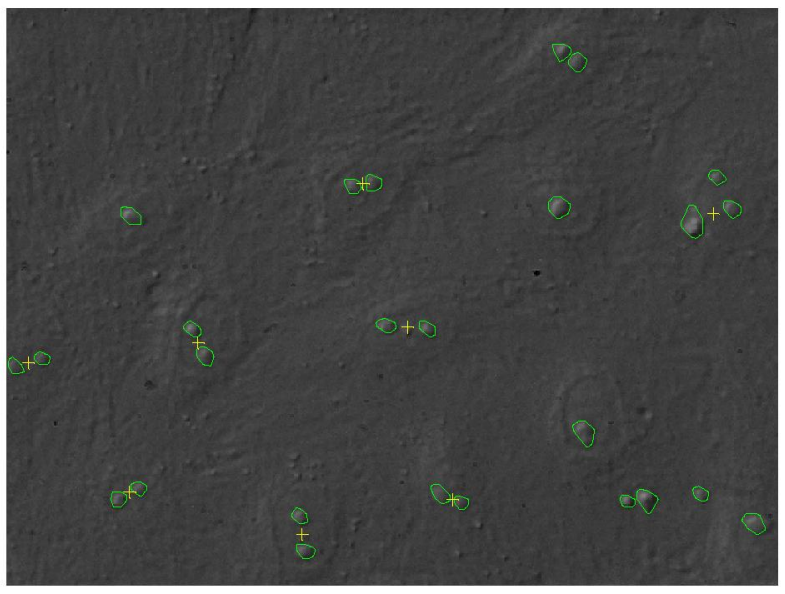

Fig 9: Applying the algorithm for detecting points for an injection site. The injection sites are denoted by a cross.

\section{CONCLUSION}

Leveraging machine vision and precise motion control, a cell injection system has been developed for endothelial cells. Image processing algorithms for the adherent cells are proposed in this paper. The algorithm for recognizing endothelial cell nucleoli uses the surface and shadow information to locate the nucleoli in the nucleus, and determines a deposition destination by Delaunay triangulation. 436 endothelial cell nucleoli were $92 \%$ correctly recognized, demonstrating the potential of an automated adherent cell injection system to be developed by 
integrating the proposed image processing algorithm, which is capable of recognizing nucleoli and determining deposition destinations inside the nucleus from the harsh pseudo-3D images.

\section{REFERENCES}

[1] Yu J, Vodyanik MA, Kim So, Jessica AB, Frane JL, Tian S, Nie J, Jonsdottir GA, Ruotti V, Stewart R, Slukvin II, Thomson JA (2007) Induced pluripotent stem cell lines derived from human somatic cells. Science 318: 1917-1920.

[2] Madsen EC, Morcos PA, Mendelsohn BA, Gitlin JD (2008) In vivo correction of a Menkes disease model using antisense oligonucleotides. Proc Natl Acad Sci USA 105: 3909-3914.

[3] Sundaram J, Mellein BR, Mitragotri S (2004) An experimental and theoretical analysis of ultrasound-induced permeabilization of cell membranes. Biophys J 87: 1013-1033.

[4] Rols MP (2006) Electropermeabilization, a physical method for the delivery of therapeutic molecules into cells. Biochim Biophys Acta 1758: 423-428

[5] Park JW (2002) Liposome-based drug delivery in breast cancer treatment. Breast Cancer Res 4: 95-99.

[6] Chavanpatil MD, Khdair A, Panyam J (2006) Nanoparticles for cellular drug delivery: Mechanisms and factors influencing delivery. J Nanosci Nanotechnol 6: 2651-2663.

[7] Sun Y, Nelson BJ (2002) Biological cell injection using an autonomous microrobotic system. Int J Robot Res 21: 861-868.

[8] Celis JE (1984) Microinjection of somatic cells with micropipettes: Comparison with other transfer techniques. Biochem J 2: 281-291.

[9] Zhao Z, Cao Y, Li M, Meng A (2001) Double-stranded RNA injection produces nonspecific defects in zebrafish. Dev Biol 1: 215-223.

[10] Scherp P, Hasenstein KH (2003) Microinjection-a tool to study gravitropism. Space Res 31: 2221-2227.

[11] Gahtan E, O'Malley DM (2003) Visually guided injection of identified reticulospinal neurons in zebrafish: A survey of spinal arborization patterns. J Comp Neurol 459: 186-200.

[12] King R (2004) Gene delivery to mammalian cells by microinjection. Methods Mol Biol 245: 167-174.

[13] Zappe S, Fish M, Scott MP, Solgaard O (2006) Automated MEMSbased drosophila embryo injection system for high-throughput RNAi screens. Lab Chip 6: 1012-1019.

[14] Viigipuu K, Kallio P (2004) Microinjection of living adherent cells by using a semi-automatic microinjection system. ATLA 32: 417-423.

[15] Wang WH, Sun Y, Zhang M, Anderson R, Langille L, Chan WCW (2008) A microrobotic adherent cell injection system for investigating intracellular behavior of quantum dots. Proc EEE International Conference on Robotics and Automation (ICRA'2008): accepted.

[16] Kumar R, Kapoor A, Taylor RH (2003) Preliminary experiments in robot/human cooperative microinjection. Proc IEEE International Conference on Intelligent Robots and Systems (IROS'2003): 31863191.

[17] Mattos L, Grant E, Thresher R, Kluckman K (2007) New developments towards automated blastocyst microinjections. Proc IEEE International Conference on Robotics and Automation (ICRA'2007): 1924-1929.

[18] Pillarisetti A, Pekarev M, Brooks AD, Desai JP (2007) Evaluating the effect of force feedback in cell injection. IEEE Transactions on Automation Science and Engineering 4: 322-331.

[19] Wang WH, Liu XY, Gelinas D, Ciruna B, Sun Y (2007a) A fully automated robotic system for microinjection of zebrafish embryos. PLoS ONE 2: e862.

[20] Wang WH, Liu XY, Sun Y (2007b) Contact detection in microrobotic manipulation. Int J Robot Res 26: 821-828.

[21] Canny JFA (1986) Computational approach to edge detection. IEEE Trans Pattern Analysis and Machine Intelligence 8: 679-698.
[22] Lowe DG (1999) Object recognition from local scale-invariant features. Proc IEEE 7th International Conference on Computer Vision: 1150-1157.

[23] Xu CY, Prince JL (1998) Snakes, shapes, and gradient vector flow. IEEE Trans Image Processing 7: 359-369.

[24] http://www.mathworks.com 